INFECTIOUS DISEASE

\section{Finding the keys to the placental barrier}

Two bacterial proteins, InlA and InlB, have essential and interdependent roles in enabling Listeria monocytogenes to cross the placental barrier and cause fetoplacental listeriosis, Oliver Disson and colleagues report in Nature.

Several organisms can breach the placental barrier with fatal consequences for a developing fetus. In the case of fetoplacental listeriosis, which is caused by L. monocytogenes,

L. monocytogenes specifically targets the placenta in vivo, if and only if both InIA and InIB pathways are functional.

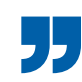

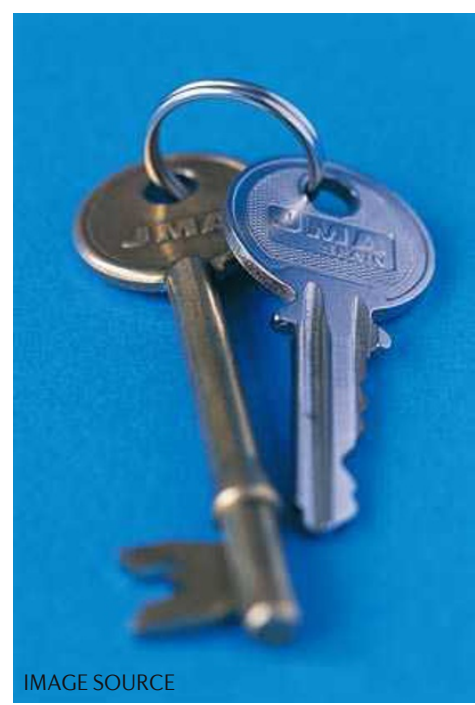

neonatal mortality is high, even when antimicrobial therapy is administered to the mother. The mechanism by which L. monocytogenes breaches the placental barrier has remained unclear, in part because L. monocytogenes infection involves different pathways in different species. Thus, although Disson et al. thought that bacterial ligands InlA, which binds human and guinea-pig E-cadherin, and InlB, which binds human and mouse Met, work together in humans to breach the placental barrier, they needed animal models that encode active E-cadherin and Met pathways to test their theory.

The authors characterized two suitable animal models: gerbil, which is a natural host for L. monocytogenes, and a knock-in mouse that constitutively expresses a humanized version of E-cadherin that was enabled to interact with InlA. To evaluate the relative contribution of InlA and InlB to fetoplacental infection, the authors used a competition index method. Gerbils and knock-in mice were coinoculated with a chloramphenicolresistant strain of L. monocytogenes and with one of four possible other strains: a control wild-type strain, an inlA knockout mutant, an inlB knockout mutant or an inlA inlB double knockout mutant. In both the gerbil and the knock-in mouse, all three knockout mutants were extremely defective in fetoplacental invasion.

On the basis of these and other experiments, the authors conclude that " $L$. monocytogenes specifically targets the placenta in vivo, if and only if both InlA and InlB pathways are functional." "To our knowledge," the authors add, "this is the first report demonstrating the speciesand tissue-specific recognition of the placenta by a microbial pathogen enabling it to breach the placental barrier." As yet, however, crucial questions remain unresolved. Why is InlA sufficent for infection at the intestinal level, but not at the placental level? How exactly do InlA and InlB function together to breach the placental barrier? Although Disson and colleagues have made crucial steps by identifying the ligandreceptor interactions that enable L. monocytogenes to breach the placental barrier, much remains to be done to find an adequate treatment strategy for fetoplacental listeriosis. Asher Mullard

ORIGINAL RESEARCH PAPER Disson, O. et al. Conjugated action of two species-specific invasion proteins for fetoplacental listeriosis. Nature 17 Sep 2008 (doi:10.1038/nature07303) FURTHER READING Hamon, M., Bierne, H. \& Cossart, P. Listeria monocytogenes: a multifaceted model. Nature Rev. Microbiol. 4, 423-434 (2006) 